(1)

CrossMark

\title{
How can we better predict pulmonary blood clots in patients hospitalised for COVID-19?
}

\author{
Laurent Bertoletti (1) ${ }^{1,2,3,4}$ and Menno V. Huisman ${ }^{5}$
}

Affiliations: 'Service de Médecine Vasculaire et Thérapeutique, CHU de St-Etienne, Saint-Etienne, France. ${ }^{2}$ INSERM, UMR1059, Equipe Dysfonction Vasculaire et Hémostase, Université Jean-Monnet, Saint-Etienne, France. ${ }^{3}$ INSERM, CIC-1408, CHU Saint-Etienne, Saint-Etienne, France. ${ }^{4}$ F-CRIN INNOVTE network, SaintEtienne, France. ${ }^{5}$ Dept of Thrombosis and Hemostasis, Leiden University Medical Center, Leiden, The Netherlands.

Correspondence: Menno V. Huisman, Dept of Thrombosis and Hemostasis, Leiden University Medical Center, Leiden, Albinusdreef 2, 2333ZA Leiden, The Netherlands. E-mail: m.v.huismandlumc.nl

@ERSpublications

D-dimer threshold at $2590 \mathrm{ng} \cdot \mathrm{mL}^{-1}$ is able to predict pulmonary embolism in COVID-19 patients with clinical deterioration https://bit.ly/3kYObc3

Cite this article as: Bertoletti L, Huisman MV. How can we better predict pulmonary blood clots in patients hospitalised for COVID-19? Eur Respir J 2020; 56: 2003092 [https://doi.org/10.1183/ 13993003.03092-2020].

The emergence of coronavirus disease 2019 (COVID-19) has put pressure on health systems around the world $[1,2]$. This coronavirus has also questioned much of our medical knowledge, with each day seeing the appearance of a new possible clinical expression of the virus [3]. Although its physiopathology is still poorly understood, the vascular tropism of the disease now seems to be a major pathway [4]. Recent studies highlight the development of a specific pulmonary vascular endothelialitis associated with thrombosis and angiogenesis [5].

A strong association between coronavirus infection and the risk of venous thromboembolism (VTE) was suggested by Chinese authors [6], who described an increase in the blood level of D-dimer (increase associated with an increased risk of death) [7]. A specific coagulopathy was evoked [8], as well as a possible increased risk of VTE. However, it also appeared that COVID-19 may also challenge our usual way to deal with VTE suspicion and management [9]. First, the respiratory impairment of COVID-19 directly impacts the usual modalities of suspicion of VTE. In patients admitted to the emergency department with a respiratory picture compatible with a COVID-19, the symptomatology close to that of pulmonary embolism (PE) may hinder the evocation of alternative diagnoses. The (now classic) secondary respiratory deterioration raises the same question of alternative diagnosis as PE. When VTE is suspected, the pandemic situation makes access to vascular and thoracic explorations more complex, requiring the development of parallel flows for contagious and non-contagious patients. In patients admitted to the intensive care unit (ICU), the issues are even more specific, with the difficulty of diagnosing VTE (and particularly PE) in sedated patients, and the technical difficulty of performing chest imaging in intubated patients with renal failure.

It is in this context in the current issue of the European Respiratory Journal that MouHAT et al. [10] report their retrospective analysis concerning the suspicion of pulmonary embolism in COVID-19 patients admitted to a university hospital in a pandemic zone.

Received: 10 Aug 2020 | Accepted: 12 Aug 2020

Copyright OERS 2020. This version is distributed under the terms of the Creative Commons Attribution NonCommercial Licence 4.0. 
The authors analysed the clinical and biological data of 349 COVID patients admitted in 1 month. Among the 162 patients who underwent a computed tomography pulmonary angiography (CTPA) because of a severe clinical presentation (respiratory rate $\geqslant 30$ breaths $\cdot \min ^{-1}$, oxygen saturation measured by pulse oximetry $\leqslant 93 \%$, or rapid clinical worsening), PE was diagnosed in $44(27.2 \%)$ patients, of which $20 \%$ were at hospital admission. Two factors were associated with the risk of PE: high D-dimer levels and lack of anticoagulant therapy. The association between PE and the lack of anticoagulant therapy was mainly supported by the inclusion of all PE patients (including PE diagnosed at admission). The authors propose a D-dimer threshold at $2590 \mathrm{ng} \cdot \mathrm{mL}^{-1}$ to predict CTPA-confirmed PE in severe COVID-19 patients, with high accuracy: AUC 0.88 (95\% CI 0.809-0.932), $\mathrm{p}<0.001$. Patients with D-dimers above the cut-off of $2590 \mathrm{ng} \cdot \mathrm{mL}^{-1}$ accounted for $36.0 \%$ (95\% CI 27.5-45.2) of the overall population, $42.6 \%$ (95\% CI 30.7-55.2) of patients in ICU, and $15.9 \%$ (95\% CI 9.2-24.9) of patients in conventional COVID wards. While their study is retrospective and as a result exposed patients to heterogeneous management, it gives us an interesting perspective.

Where do we go from here? First, we need to prospectively assess the frequency of PE at admission and the validation of the D-dimer cut-off of $2500 \mathrm{ng} \cdot \mathrm{mL}^{-1}$ to indicate PE to be present in COVID-19 patients. The current work suggests that only one in five PE was diagnosed at admission, which is lower than in another study [11], but in agreement with a recent retrospective study [12]. It also confirms that D-dimers still have a role in the stratification of a patient prognosis, being associated with an increased risk of death ( $>1000$ ) but also PE (if $>2500$ ). The combination of D-dimer results with a clinical prediction rule (as the Geneva [13], the Wells [14], or the YEARS scores [15] proposed in the European guidelines [16]) is still under debate and this also needs prospective validation. Secondly, we need to determine factors associated with hospital-acquired VTE in COVID-19 patients. In the current work by MouHAT et al. [10], most of the PE occurred after admission. Thromboprophylaxis is indicated in any patient admitted for COVID-19 [8], and many authors proposed to increase the dosage of usual parenteral drugs, with the hope to decrease the rate of venous and arterial complications [17], without increasing the bleeding risk. Hence, the risk and benefits of such modified thromboprophylaxis, as well as the potential of pharmacological agents targeting thromboinflammation [18], deserve further analysis.

Conflict of interest: L. Bertoletti reports grants and non-financial support for meeting attendance from Bayer, grants, personal fees for advisory board work and non-financial support for meeting attendance from BMS/Pfizer, personal fees for advisory board work and non-financial support for meeting attendance from Leo Pharma and Aspen, outside the submitted work. M.V. Huisman reports grants and personal fees from ZONMW, Boehringer Ingelheim, Bayer Health Care, Pfizer-BMS and Leo Pharma, during the conduct of the study.

\section{References}

1 Guan W, Ni Z, Hu Y, et al. Clinical characteristics of coronavirus disease 2019 in China. N Engl J Med 2020; 382: $1708-1720$

2 Zhou F, Yu T, Du R, et al. Clinical course and risk factors for mortality of adult inpatients with COVID-19 in Wuhan, China: a retrospective cohort study. Lancet 2020; 395: 1054-1062.

3 McGonagle D, O’Donnell J, Sharif K, et al. Immune mechanisms of pulmonary intravascular coagulopathy (PIC) in COVID-19 pneumonia. Lancet Rheumatol 2020; 2019: 1-9.

4 Gupta A, Madhavan M V, Sehgal K, et al. Extrapulmonary manifestations of COVID-19. Nat Med 2020; 26 $1017-1032$.

5 Ackermann M, Verleden SE, Kuehnel M, et al. Pulmonary vascular endothelialitis, thrombosis, and angiogenesis in Covid-19. N Engl J Med 2020; 383: 120-128.

6 Tang $\mathrm{N}$, Bai $\mathrm{H}$, Chen $\mathrm{X}$, et al. Anticoagulant treatment is associated with decreased mortality in severe coronavirus disease 2019 patients with coagulopathy. J Thromb Haemost 2020; 18: 1094-1099.

7 Tang N, Li D, Wang X, et al. Abnormal coagulation parameters are associated with poor prognosis in patients with novel coronavirus pneumonia. J Thromb Haemost 2020; 18: 844-847.

8 Thachil J, Tang N, Gando S, et al. ISTH interim guidance on recognition and management of coagulopathy in COVID-19. J Thromb Haemost 2020; 18: 1023-1026.

9 Bertoletti L, Couturaud F, Montani D, et al. Venous thromboembolism and Covid-19. Respir Med Res 2020; 78: 100759.

10 Mouhat B, Besutti M, Bouiller K, et al. Elevated D-dimers and lack of anticoagulation predict PE in severe COVID-19 patients. Eur Respir J 2020; 56: 2001811.

11 Poissy J, Goutay J, Caplan M, et al. Pulmonary embolism in COVID-19 patients: awareness of an increased prevalence. Circulation 2020; 142: 184-186.

12 Freund Y, Drogrey M, Miró Ò, et al. Association between pulmonary embolism and COVID-19 in ED patients undergoing CTPA: the PEPCOV international retrospective study. Acad Emerg Med 2020; 27: 811-820.

13 Le Gal G, Righini M, Roy P-M, et al. Prediction of pulmonary embolism in the emergency department: the revised Geneva score. Ann Intern Med 2006; 144: 165-171.

14 Anderson DR, Kovacs MJ, Kovacs G, et al. Combined use of clinical assessment and d-dimer to improve the management of patients presenting to the emergency department with suspected deep vein thrombosis (the EDITED Study). J Thromb Haemost 2003; 1: 645-651.

15 van der Hulle T, Cheung WY, Kooij S, et al. Simplified diagnostic management of suspected pulmonary embolism (the YEARS study): a prospective, multicentre, cohort study. Lancet 2017: 6736. 
16 Konstantinides S V, Meyer G, Becattini C, et al. 2019 ESC Guidelines for the diagnosis and management of acute pulmonary embolism developed in collaboration with the European Respiratory Society (ERS): The Task Force for the diagnosis and management of acute pulmonary embolism of the European Society of Cardiology (ESC). Eur Respir J 2019; 54: 1901647.

17 Klok FA, Kruip MJHA, van der Meer NJM, et al. Incidence of thrombotic complications in critically ill ICU patients with COVID-19. Thromb Res 2020: 100632.

18 Bikdeli B, Madhavan M V, Gupta A, et al. Pharmacological agents targeting thromboinflammation in COVID-19: review and implications for future research. Thromb Haemost 2020; 120: 1004-1024. 